\section{Other riffs on cooperation are already showing how well a wiki could work}

SIR - Barend Mons's Wiki for Professionals at www.wikiprofessional.info is among the first open collaborative databases to use the wiki format in biology, as your News story "Key biology databases go wiki" (Nature 445, 691; 2007) points out.

However, other, non-wiki resources have already shown the feasibility of cooperative, online database construction. One such success story is GeneRIF (www.ncbi.nlm. nih.gov/projects/GeneRIF), which is like a miniature wiki where the author is restricted to a single short sentence. Currently, GeneRIF contains close to 200,000 entries, and each is attached to a particular gene at the Entrez database of the National Center for Biotechnology Information.

Being interested in gene-disease relationships, we assessed the coverage and specificity of GeneRIF and compared them to OMIM (Online Mendelian Inheritance in Man), a traditional source of gene-disease information. We found that GeneRIF already covers more than twice the number of diseases per gene and includes many more newly discovered mappings (www.basic.northwestern.edu/ publications/generifdo). This seems to us to answer the scepticism that has been expressed about the expected community involvement in wiki collaborations.

John D. Osborne ${ }^{\star}$, Simon Lin't,

Warren A. Kibbe

*University of Alabama at Birmingham, 845 19th Street South, Bevill Building,

Room 273C, Birmingham, Alabama 35294, USA $\uparrow$ Robert H. Lurie Comprehensive Cancer Center, Northwestern University, 676 North St Clair, Suite 1200, Chicago, Illinois 60611, USA †Center for Genetic Medicine,

Robert H. Lurie Comprehensive Cancer Center and Feinberg School of Medicine, Northwestern University, 676 North St Clair, Suite 1200, Chicago, Illinois 60611, USA

\section{Law and research could add up to profitable niche drugs}

SIR - In response to your Editorial "A changing drug supply" (Nature 445, 460; 2007), W. Ross Tracey points out in Correspondence that niche drugs may be just as expensive as blockbuster drugs to bring to the market, but a pharmaceutical company's revenue from them is likely to be only a fraction of what it can earn from selling blockbusters ("Niche drugs aren't a cheap alternative to blockbusters"

Nature 445, 818; 2007).

This is not necessarily so, in my opinion.
Many drugs fail in clinical development, and some drugs have to be withdrawn after causing severe side effects in some patients. Once it becomes possible to determine what causes these patients to show adverse reactions, it may become feasible to pre-select a smaller patient population that will only respond positively and not suffer any adverse events. Although this strategy may not deliver blockbuster drugs, it could considerably reduce the present high attrition rate in drug development and thus make niche drugs economically viable.

Another point is that the US Food and Drug Administration uses financial incentives and an accelerated review process to support the development of drugs for 'orphan diseases' - conditions so uncommon that drug companies would otherwise have no incentive to seek cures for them (see the Orphan Drug Act, www. fda.gov/orphan/oda.htm).

\section{Burkhard Haefner}

Johnson \& Johnson Pharma Research and

Development, Turnhoutseweg 30,

Box 6423, 2340 Beerse, Belgium

\section{Why do so few women speak at science meetings?}

SIR - Mary Ann Holmes and Suzanne O'Connell comment on the lack of women in the academic ranks in your Recruiters article "Leaks in the pipeline" (Nature 446, 346; 2007). In the same issue, advertisements for two Nature conferences illustrate part of the problem - the poor representation of women speakers at scientific meetings.

The Nature conference "Oncogenes and human cancer: the next 25 years" features 36 speakers, of whom four are women. The "Days of molecular medicine: emerging technologies and cancer biology" conference, co-sponsored by Nature Medicine, features 19 speakers, of whom two are women. There are many accomplished women scientists in the areas covered by these meetings. There is no obvious reason why the number of women speakers should be so low.

The representation of women speakers at many meetings remains dismally poor and thus may contribute to the lack of success of women in academia.

However, this is a problem that could be easily remedied, if more attention were paid by organizers and the agencies that provide funding for meetings to the issue of whether qualified female speakers have been missed.

Pamela A. Silver

Department of Systems Biology,

Harvard Medical School,

Boston, Massachusetts 02115, USA

\section{Who will start the 3 Rs ball rolling for animal welfare?}

SIR - Hanno Würbel, in his Correspondence "Publications should include an animalwelfare section", suggests an effective and powerful way in which journals, by including a dedicated category for the $3 \mathrm{Rs}$ - replace, refine, reduce - in the methodology section, could benefit both scientific research and animal welfare (Nature 446, 257; 2007, and see http://blogs.nature.com/nautilus/2007/ 03/proposal_for_journals_to_inclu.html) .

Ever since this idea was recommended by the Nuffield Council on Bioethics, I have been probing scientists for their response to it. Almost without exception, those I have approached have recognized its potential and support the idea verbally. However, they have consistently been reticent about providing any written endorsement.

The first, but, in my opinion, least likely, reason for this behaviour could be apathy, because this sensible, moderate and pragmatic proposal does not arouse the same emotions as the 'animal research' debate itself.

Second, the polarized nature of this topic may foster the fear that publicly supporting any measure to improve animal welfare will be perceived as a defection by other scientists.

Third, there may be an unwillingness to admit what some would see as a weakening of stance - the thin end of a wedge that threatens to phase out animal research altogether. Although my personal desire is that this would indeed be the outcome, the evidence speaks otherwise. Despite the best efforts of animal-welfare advocates since the 3Rs concept was first introduced, 'replacements' have become established only when they are of scientific benefit so it seems unreasonable that efforts to encourage them should be viewed as a threat to progress.

Finally, there is resistance to going against the grain. If a single high-impact journal were to take a unilateral decision to implement this proposal and embrace the $3 R$ s as integral and essential elements of good experimental design, I believe that other editors and scientists everywhere would be happy to follow.

\section{Victoria Buck}

Division of Stem Cell Biology and

Developmental Genetics,

MRC National Institute for Medical Research, Mill Hill, London NW71AA, UK

Comments are welcome at Nautilus, the blog for authors, at the URL above.

Contributions to Correspondence may be submitted to correspondence@nature. com. They should be no longer than $\mathbf{5 0 0}$ words, and ideally shorter. 